\title{
Civilisations
}

Revue internationale d'anthropologie et de sciences

humaines

$54 \mid 2006$

Expériences de recherche en République

démocratique du Congo

\section{Pour une analyse du discours des Eglises de réveil à Kinshasa}

Méthode et contexte

\section{Alexis Matangila}

\section{(2) OpenEdition}

\section{Journals}

Édition électronique

URL : http://journals.openedition.org/civilisations/343

DOI : $10.4000 /$ civilisations.343

ISSN : 2032-0442

Éditeur

Institut de sociologie de l'Université Libre de Bruxelles

Édition imprimée

Date de publication : 1 avril 2006

Pagination : 77-84

ISBN : 2-87263-006-6

ISSN : 0009-8140

\section{Référence électronique}

Alexis Matangila, « Pour une analyse du discours des Eglises de réveil à Kinshasa », Civilisations [En

ligne], 54 | 2006, mis en ligne le 01 avril 2009, consulté le 19 avril 2019. URL : http://

journals.openedition.org/civilisations/343; DOI : 10.4000/civilisations.343 


\title{
Pour une analyse du discours des Eglises de réveil à Kinshasa
}

\author{
Méthode et contexte
}

\author{
Alexis MATANGILA
}

Résumé : Fréquentées par une population relativement jeune qui fait face aux difficultés d'une société congolaise économiquement malade, les Eglises de réveil constituent un espace de circulation d'un discours religieux particulier. Les discours de leurs pasteurs sont remplis de promesses quant au salut de l'âme, à la santé du corps, à la protection contre les mauvais esprits et au bien-être matériel. Ce discours assure ainsi des fonctions messianique, prophétique et thérapeutique au sein de la société. Deux approches sont complémentaires pour étudier cet espace de langage. L'approche sociologique renseigne sur l'univers social qui entoure le discours des adeptes : âge, sexe, province d'origine, degré d'instruction, religion d'origine, raison du changement de religion, profession, etc. La deuxième approche est pragmatique : elle entreprend, grâce à la construction d'une grille aux catégories méticuleusement choisies, l'étude des signes linguistiques constituant les textes d'analyse en rapport avec leurs utilisateurs.

Mots-clés : analyse de discours, Eglises de réveil, communauté de langage, linguistique pragmatique, Kinshasa.

Summary: Revival churches have become important spaces for the circulation of religious discourse amongst youth in an economically sick Congo. Their preachers promise salvation, health, protection from evil spirits and material well-being: messianic, prophetic and therapeutic messages are intertwined. Two approaches are complementary in the study of these language spaces. The sociological approach (gathering and analysing data about age, sex, place of origin, education, first religion, reason for changing religion, profession, etc.) helps understand the social universe that contextualises the adepts' discourses. The second approach is pragmatic: thanks to a carefully chosen framework of categories, it studies the linguistic signs constituting the texts being analysed in relation to their users.

Key words: discourse analysis, revival churches, community of language, pragmatic linguistics, Kinshasa. 


\section{Le contexte d'émergence des Eglises de réveil}

$\mathrm{D}_{\mathrm{r}-1}$ epuis près de deux décennies, il s'observe une recrudescence du phénomène religieux à Kinshasa. Le christianisme s'est enrichi d'un nouvel espace de discours - les Eglises de réveil - qui a provoqué des bouleversements dans les pratiques langagières. En août 2004, nous avons réalisé un travail intitulé «Les enjeux de la communication dans les Eglises de réveil à Kinshasa. Essai d'analyse d'un discours religieux en milieu diglossique » (Matangila 2004). L'hypothèse de base était l'existence des pratiques langagières nouvelles et spécifiques qui organisent ce nouveau discours religieux. Nous avons postulé que les Eglises de réveil sont un grand espace de discours qui détient, d'une part, un type spécifique d'énoncés linguistiques et de néologismes qui isole tout un univers sémantique et, d'autre part, une idéologie propre et à partir de laquelle elles rejettent les credo des autres Eglises, les déclarant non conformes aux textes bibliques. Elles ont élaboré leurs propres compétences et normes sociolinguistiques, un code de l'imaginaire discursif qui thématise leurs doctrines et qui connaît une réelle vitalité linguistique.

L'analyse du discours des Eglises de réveil nous a conduit à rechercher d'abord les causes et les contextes de leur émergence. L'étude avait postulé un lien de causalité entre les «pratiques mentales » du colonisateur et la réaction du colonisé pour se défaire du carcan de l'occupation. Le discours des premières Eglises indépendantes avait au départ une fonction messianiste : promettre aux adeptes une patrie propre à eux, dirigée par euxmêmes, loin des avatars du colonialisme et de son christianisme ambigu. Mais à partir de l'indépendance du Congo, le déferlement de ces Eglises indépendantes s'explique par d'autres causes : la crise économique, les maladies incurables, l'insécurité sociale, les problèmes de sorcellerie, le manque de repères sûrs, la loi de la jungle, l'incertitude du lendemain, etc. A la manière des gnoses de tous les temps difficiles, ces Eglises élaborent un « discours solution » qui promet des lendemains meilleurs aux adhérents et qui mobilise les masses. Les anciennes Eglises (catholique et protestante) se vident en leur faveur.

Avec le discours des Eglises de réveil, le christianisme kinois est sérieusement mis à l'épreuve. Les veillées mortuaires, les célébrations de noces, les réunions de famille, les rencontres amicales ou politiques, etc. sont devenues propices à la prière. L'euphorie religieuse suscitée par ces Eglises a profondément marqué les pratiques langagières des Kinois : " le langage de tous pue la religion!", « le discours des Kinois est empreint d'une religiosité superstitieuse ! », « Kinshasa n'a que le nom de Jésus dans la bouche ! », dénoncent nos enquêtés. Les fragments linguistiques «sans effet», «gloire à Dieu », «c'est démoniaque », « esprit de mort», " esprit d'impudicité », etc. qui sont à présent fréquents dans le langage des Kinois attestent des mutations culturelles réelles intervenues dans notre société. Le linguiste ne peut pas rester indifférent à ces émergences.

\section{Description de la méthode}

Notre démarche s'est divisée en deux grandes phases, dont la première visait la collecte des données. Une enquête menée à l'échelle de Kinshasa avait révélé la présence de plus de trois mille groupes de prières ou Eglises de réveil. Seules 391 étaient régulièrement enregistrés par la Ligue des Eglises et Ministères de Réveil au Congo. Nous avons soigneusement prélevé dans ce lot 39 Eglises, soit $10 \%$ du total, disséminées à travers les 24 communes de Kinshasa. 
Après identification de ces 39 lieux d'enquête, un questionnaire de pré-enquête fut conçu et distribué à raison de dix formulaires par Eglise. La pré-enquête devait nous aider à connaître les difficultés éventuelles que pouvaient éprouver nos enquêtés à répondre à nos questions. Effectivement, nous avons dû reformuler notre questionnaire avant de le redistribuer pour l'enquête proprement dite. Des 975 questionnaires distribués dans les Eglises, 741 ont été remplis et renvoyés par des fidèles. L'enquête visait surtout à connaître l'âge et le sexe des adeptes enquêtés, leur niveau d'instruction, leur province d'origine, leur commune de résidence, leur religion d'origine, leurs opinions sur les Eglises de réveil, les raisons de leur engouement massif vers celles-ci et le bénéfice obtenu du changement de religion. Après dépouillement, il est apparu qu'elles sont fréquentées par une population en majorité jeune, à prédominance féminine, relativement instruite, qu'on retrouve dans toutes les communes, originaire (dans l'ordre décroissant) des provinces du Bas-Congo, du Bandundu et des deux provinces de Kasaï. La majorité des fidèles sont issus de l'Eglise catholique : c'est le cas de 533 des 741 informateurs, dont seulement 129 étaient issus de l'horizon protestant.

Nous reproduisons ici quelques réponses dans leur version d'origine pour que le lecteur puisse se faire une idée de leur diversité quant au contenu et des formulations écrites auxquelles est confronté le chercheur lors d'une enquête par questionnaire. A la question « Que pensez-vous de vos Eglises appelées sectes? », nous avons reçu plusieurs réponses dont «Elles vous invitent à vous joindre à nous », " Nous apprenons la vérité que les otre eglise refise d'ansegné », «manque d'organisation, trop de querelles de leadership », « elles sont la lumière du monde », etc. A la question « Pourquoi avez-vous changé d'Eglise? », nous avons obtenu beaucoup de réponses dont « Les autres Eglises n'ont pas guéri ma femme du cancer de sein qui avait failli l'emporter », " J'avait besoins de delivranse », «Sir lordre di Saint-Esprit », «à la demande de mon fiansé », « ça ne te regarde pas », etc. A la question «Qu'avez-vous obtenu de Dieu en récompense de votre changement de religion? », les réponses n'ont pas manqué : "j’ai sui nez de nouvau!», «Ma femme est guérie du cancer de sein après avoir reçu Jésus comme son Sauveur personnel et après qu'on ait prié pour elle avec imposition des mains. N'est-ce pas merveilleux? », « nou conessson la verite », « je suis dévénue la fille de Dieu selon Jean $1: 12 »$, « Beaucoup de bénédictions sans nombre », « Mes rhumatismes ont disparu. Alors si ton Dieu est mort et ne fait rien pour toi, essayez le mien », « je suis certain de mon salut », etc.

Ensuite, durant cette enquête, nous avons observé seize genres discursifs pendant le culte et en dehors de celui-ci : le chant, la prière, l'instruction, l'appellatif, la récitation, le communiqué, le dialogue, la lecture, la bénédiction, la salutation, la causerie, le témoignage, les cris de slogan, l'enseignement, la confession, la prophétie. Toutefois, ne pouvant pas envisager l'étude de tous ces genres dans le seul cadre de ce travail, notre recherche s'est limitée à quatre d'entre eux : la prière, la prophétie, le témoignage et l'enseignement.

Au cours de cette même enquête dans les 39 Eglises retenues, nous avons enregistré 43 messages prophétiques énoncés pendant les cultes. Nous avons aussi récolté 96 messages écrits dans les Eglises qui ont leurs journaux ou leurs revues propres. A partir de ces 139 textes, un corpus fort de douze textes méticuleusement sélectionnés a été élaboré. Les textes modèles retenus, à savoir trois prières, trois prophéties, trois témoignages et trois enseignements, sont de longueur inégale. Ils ont été choisis en fonction de la richesse des stratégies langagières mises en œuvre par les locuteurs pour convaincre les auditeurs. 
Après cette phase de récolte des données d'enquête, nous sommes passé à celle de l'analyse pragmatique du discours. Nous avons soumis ces douze textes à une même grille d'analyse dont les catégories s'appuyaient sur les données pragmatiques de la langue. Ces dernières, en effet, permettent de relever tous les fragments linguistiques qui prennent des fonctions particulières pendant leur énonciation. Notre grille d'analyse était forte de plusieurs catégories : le cadre figuratif, le posé et le présupposé, les fonctions linguistiques prédominantes, les intentions énonciatives, le référent du discours, les actes de discours, les indices de contextualisation, les procès rhétoriques présents dans le discours. Nous étudierons plus particulièrement quatre d'entre elles dans la suite de ce texte.

\section{Le cadre figuratif}

Cette catégorie analytique identifie les protagonistes de chaque pôle du procès communicatif (qui parle à qui ?), et la relation de rôle qui les gère (pourquoi se parlent-ils ainsi ?). Est donc évoquée ici la notion centrale de " place », c'est-à-dire du rôle prescrit à chaque interlocuteur par le discours. En effet, toute parole est émise d'une "place » et convoque l'interlocuteur à une autre "place» qui lui est corrélative. Parler en tant que pasteur (ou en tant que conducteur spirituel), c'est assigner à l'autre la " place» de fidèle; et c'est aussi se faire accepter comme tel par l'interlocuteur. Mais ces «places» ne sont pas des données sociolinguistiques stables, irréfutables. Il arrive en effet que les protagonistes se mettent à négocier les rapports de « place » au cours d'un même procès de parole.

Le cadre figuratif définit en même temps les participants ratifiés et les simples témoins $\mathrm{du}$ procès communicatif. A Kinshasa, en effet, où il y a souvent des tensions entre le discours religieux et le discours politique, les homélies télévisées des pasteurs retentissent souvent bien au-delà des parvis de l'église. En février 2004, l'archibishop Kutino Fernando se faisait interviewer sur l'affaire « Sauvons le Congo ». Le vrai destinataire de son message n'était pourtant pas l'intervieweur, mais le Gouvernement de la République. Et en réponse au message, le Gouvernement réagit immédiatement en fermant l'église du pasteur Kutino mis en fuite. Cette catégorie analytique est donc indispensable car elle aide à connaître le vrai destinataire du message linguistique émis par le destinateur, et, par conséquent, à faciliter la compréhension du fonctionnement du discours étudié.

\section{Les intentions énonciatives}

Il n'existe pas de parole neutre. Dans chaque événement de parole, le locuteur invite l'interlocuteur à faire quelque chose, c'est-à-dire à adopter un comportement. Il y arrive moyennant plusieurs stratégies discursives. Par exemple, depuis les pillages du début des années 1990, une crise économique sans précédent sévit en RDC, mettant à l'épreuve la foi de beaucoup de chrétiens. La misère, le désespoir frappent : les Kinois comptent les coups. C'est ainsi que lors de notre enquête en 1998 dans une Eglise de réveil à Lemba, le pasteur proposa une piste de salut pour ses fidèles dans une prédication inspirée du premier livre des Rois (7 : 9-16). L'extrait relate l'histoire d'une veuve de Sarepta. Cette dernière avait offert au prophète Elie, au plus fort de la grande famine qui avait sévi en Israël en ces temps-là, un gâteau fait du peu de farine et d'huile qui restaient encore pour sa propre subsistance et celle de son fils. Et grâce à la prière du prophète, la farine et l'huile ne manquèrent plus dans la maison de la veuve jusqu'à la fin de la période de la famine. Après un galvanisant commentaire de cet extrait, tous les fidèles étaient conviés 
à se lever pour expérimenter le miracle vécu par la veuve. Le pasteur les exhortait à consentir chacun une offrande du prophète, c'est-à-dire à offrir à Dieu, via lui-même (le pasteur), ce qu'ils avaient de plus précieux pour leur subsistance pendant ces jours de crise. En récompense, le pasteur prierait pour leur faire obtenir du ciel une abondance qui ne tarirait point.

En tenant ce discours de prospérité et de salut social, l'intention apparente de l'orateur était d'aider les adeptes en leur présentant une piste d'enrichissement rapide, saine et biblique. Mais ses intentions énonciatives réelles et cachées étaient, certainement, de se mettre lui-même à l'abri des besoins en soutirant de l'argent et des biens aux adeptes naïfs. De même, en rappelant inlassablement aux fidèles les versets bibliques du genre « Donnez, et il vous sera aussi donné dans une mesure qui déborde », «Tu ne te présenteras pas devant l'Eternel ton Dieu les mains vides »-versets bibliques cités dans presque chaque Eglise lors de notre enquête -, les pasteurs affichaient l'intention apparente de rappeler simplement des recommandations bibliques aux fidèles, mais les visées réelles de leur discours étaient, soit de doter l'Eglise des moyens financiers pour son fonctionnement, soit de s'enrichir sur le dos des fidèles naïfs.

\section{Les actes de discours}

Quelles sont les différentes actions que les locuteurs posent dans les discours qu'ils tiennent? Que font Dieu, les conducteurs spirituels et les fidèles lorsqu'ils produisent telle ou telle parole? Plusieurs typologies d'actes de langage existent, dont principalement celles des philosophes anglais Austin (1962) et Searle (1972). Nous avions préféré travailler avec celle de Searle pour des raisons de clarté et d'applicabilité. Ce philosophe classe toutes les productions langagières en cinq grandes familles d'énoncés que nous présentons ici.

Les énoncés assertifs sont ceux dont le propos engage la responsabilité du locuteur sur l'existence d'un état de choses, sur la vérité de la proposition exprimée. Quand un locuteur énonce assertivement un propos, la direction d'ajustement va toujours des mots au monde, ses paroles devant se conformer à la réalité qu'elles évoquent. Lorsque, dans le troisième texte prophétique sélectionné par notre enquête, Dieu lui-même parla aux fidèles de l'Eglise Béthel de l'apôtre Emmanuel Mbiye, à Limete, par la bouche d'une femme prophétesse et leur dit: «Je suis Jéhovah trois fois Saint!», il n'avait fait qu'asserter quelque chose : sa nature divine qui est tout à fait sainte.

Les énoncés déclaratifs sont ceux dont l'accomplissement heureux provoque toujours la mise en correspondance du contenu propositionnel avec la réalité. La direction d'ajustement va donc du monde aux mots, la réalité nommée par le locuteur étant appelée à se conformer à sa parole. Ce sont des énoncés linguistiques qui ne décrivent plus les états de choses, ils les modifient plutôt, comme lorsqu'au cours d'une de nos enquêtes à Limete, à l'occasion d'un mariage, le pasteur dit « Jean-Paul et Madeleine, je vous déclare à présent mari et femme !» : il avait posé un acte qui modifiait les états civils de ces deux personnes. Il avait donc produit un énoncé déclaratif ou performatif. Mais cette modification ne serait pas advenue si l'énonciateur n'était pas socialement investi d'un pouvoir de réguler ces genres de choses.

Les énoncés directifs sont des impératifs. La fonction linguistique incitative est dominante : l'interlocuteur. Ce dernier est sommé de se conformer au dire du locuteur. C'est pourquoi, la direction d'ajustement va du monde aux mots. C'est le cas de l'impératif 
« Cessez vos bruits ! » prononcé par Dieu (à travers la prophétesse qu'il avait investie de sa puissance), dans le troisième texte prophétique sélectionné. Les adeptes qui avaient exécuté cet ordre divin n'avaient fait qu'adapter leur comportement à la parole de Dieu.

Les promissifs sont des énoncés qui engagent le locuteur dans un rapport d'obligation vis-à-vis de l'interlocuteur. Quand, dans le premier texte prophétique sélectionné, Jésus dit à un adepte apparemment incrédule : «Exécute la parole de mon serviteur, tu verras ma gloire. Alléluia ! », il lui avait fait une promesse qu'il était obligé d'accomplir. Comme on le voit, la direction d'ajustement va du monde au mot, la chose promise devant se conformer à la parole prononcée.

Les expressifs constituent une classe d'énoncés sans direction d'ajustement. Quand, lors notre enquête, les pasteurs saluaient les adeptes, disant : « Je vous salue tous au nom de Jésus ! », ils n'assertaient rien, ne modifiaient en rien les états des adeptes, ne leur donnaient aucun ordre à exécuter et ne leur faisaient aucune promesse. Leur parole ne se conformait ni aux chrétiens dans l'auditoire, ni à la salutation : ils énonçaient seulement un expressif, c'est-à-dire qu'ils exprimaient dans un contenu propositionnel, leur propre état psychologique spécifié dans la condition de sincérité.

\section{Mode d'énonciation}

Cette catégorie rend compte de la manière dont le locuteur peut surgir dans son énoncé et lui donner les marques de sa personne, d'une part, et de la manière dont il peut complètement faire disparaître les indices d'énonciation, pour donner à sa parole la force de l'évidence. Il rend aussi compte de la manière dont ce même locuteur peut, dans son discours, soit référer à des faits appartenant à un passé quelque peu révolu, soit référer aux faits récents ou directement liés à l'instance même de l'énonciation. Nous avions au départ repris à Bronckart (1985: 63) l'hypothèse de l'existence de trois modes de discours liés à trois modes d'énonciation : narratif, discursif et théorique.

Le mode d'énonciation narratif s'applique à la parole d'un locuteur qui réfère à des faits relevant d'un passé révolu. Le système temporel de telles paroles s'organise autour du passé simple, du plus-que-parfait et de l'imparfait, comme ce texte de témoignage recueilli lors de nos enquêtes et intitulé « Tirée de la poubelle » l'illustre :

Pour des raisons d'études, mes parents (...) avaient préféré me laisser à Kinshasa.

Tout allait bien jusqu'au jour où je commençais à souffrir des maux de tête. J'avais minimisé la maladie au début. Mais elle s'accentuait au fur et à mesure que les jours passaient. Aucun hôpital de Kinshasa ne pouvait me guérir. C'est alors qu'une dame de mon quartier m'avoua que son mari traitait cette sorte de maladie avec beaucoup de facilité. Le jour où je me présentai chez lui, il m'accueillit avec empressement et m'introduisit dans une pièce qu'il appelait «laboratoire». Le décor m'effraya; mais le féticheur me calma en me rassurant qu'aucun malheur ne m'arriverait. Il me remit une feuille de papier sur laquelle je repris toute mon identité. Il prit la feuille, la plia soigneusement et la déposa sur la table en face. Je ne saurai jamais expliquer comment le papier disparût de la table, mais la réponse était là, cinq minutes après, accompagnée d'un paquet. Ce dernier contenait une poudre que je devais diluer dans l'eau et boire selon les consignes données dans la note réponse. C'était signé Belzébul, nom du «Grand-Maître» à qui je venais d'écrire!

Le mode d'énonciation discursif est assigné à tous les genres discursifs « où quelqu'un s'adressant à quelqu'un d'autre s'énonce comme locuteur et organise ce qu'il dit dans 
la catégorie de la personne » (Benveniste 1966 : 242). Les marques de cette propriété discursive dans le texte sont d'abord ses trois temps fondamentaux : le présent, le futur et le parfait. Mais il faut aussi ajouter l'imparfait qui est commun à toutes ces trois sous-catégories de mode d'énonciation. On pourrait associer à ces marques de temps « toutes les formes personnelles du verbe, aussi bien je/tu que il » (Benveniste 1966: 242). Le caractère linguistique de ce critère de mode d'énonciation est donc d'ordre morpho-lexicologique, dans la mesure où il impose la présence ou non des éléments morphologiques dont le sens n'est donné qu'en contexte (je, tu, mon, hier...). Nous pouvons remarquer le fonctionnement de ce mode d'énonciation en examinant cet extrait d'un texte de prière intitulée « Proclamation contre le démon de l'impudicité » :

Je suis aujourd'hui spirituellement fiancée à un seul époux, Jésus-Christ, pour être présentée à lui comme une vierge pure. Je m'attache à Jésus-Christ pour être avec lui un seul esprit. Je hais et je fuis l'impudicité. Quelque autre péché qu'un homme commette, ce péché est hors du corps, mais celui qui se livre à l'impudicité pèche contre son propre corps. Or Dieu ne m'a pas appelée à l'impudicité, mais à la sanctification. Ce que Dieu veut maintenant est que je m'abstienne de l'impudicité spirituelle et physique et que je sache posséder mon corps et mon esprit dans la sainteté et l'honnêteté (...) Je sais que mon corps est le temple du Saint-Esprit qui est en moi et que j'avais reçu de Dieu. Je ne m'appartiens point à moi-même. Ayant été rachetée à grand prix, je dois donc glorifier Dieu dans mon corps et dans mon esprit qui appartiennent à Dieu. Une femme mariée est liée par la loi à son mari tant qu'il est vivant. Si donc du vivant de son mari, elle devient la femme d'un autre homme, elle sera appelée adultère. Or Jésus-Christ avec qui je suis devenue un seul esprit est vivant. Je refuse donc toute présence insolite de quelque nature que ce soit qui voudrait me faire violer la loi de Dieu.

Le mode d'énonciation théorique est une propriété du discours qui se caractérise par son indépendance à l'égard d'une situation d'énonciation particulière (Bronckart 1985 : 63). Il fait disparaître tous les déictiques ${ }^{1}$ en surface de texte et fait comme si « les événements se racontaient d'eux-mêmes » (Benveniste 1966 : 241). La parole est dite à la troisième personne, comme dans cet extrait d'un texte d'enseignement intitulé « L'occultisme» :

L'occultisme est un système philosophique, une manière de penser, un mode de raisonnement particulier, original, qui a pour but de percer le mystère de l'origine du monde, celui de l'existence de l'homme et de Dieu. Il prétend y parvenir en se servant des connaissances que pourraient lui fournir les religions et les philosophies antiques. A ce titre, l'occultisme se propose donc comme un système philosophique complet. Il veut être à la fois une science, un art, une philosophie, une religion.

\section{Conclusion}

L'analyse pragmatique des douze textes présélectionnés a suffisamment mis en évidence des traits spécifiques communs aux discours de ces jeunes Eglises. Notre hypothèse de base postulant l'existence des pratiques langagières nouvelles et spécifiques s'est vérifiée : les Eglises de réveil se sont effectivement révélées être une communauté

1. On désigne par ce terme les pronoms de première et de seconde personne, certains adverbes de temps comme maintenant, aujourd 'hui, hier, demain et certains adverbes de lieu comme ici, là. Il est pratiquement impossible de leur attribuer une signification lorsqu'on ignore la situation d'énonciation. 
de langage possédant sa grammaire du discours et ses pratiques énonciatives spécifiques qui organisent le fonctionnement des productions langagières à l'intérieur des genres discursifs.

Ainsi, dans les trois textes relevant du genre «prière », trois moments énonciatifs se sont avérés itératifs : la dévotion, le combat et le remerciement. Les orants s'énoncent invariablement comme des brebis de Christ, et à ce titre, ils sont en proie aux forces du mal. Mais grâce à la force adjuvante du ciel, ils combattent le diable et ses acolytes pour se libérer de leur oppression. L'étape de la dévotion privilégie des actes de langage de type assertif; les combats recherchent plutôt les directifs et les déclaratifs, tandis que le remerciement recourt aux expressifs, aux assertifs voire aux directifs. A chaque étape dévotive, l'orant se décrit comme un être faible, quémandeur de force adjuvante, campant en face d'un être infiniment puissant. A l'étape de combat, il devient un être fort au physique comme au moral, et assigne à l'interlocuteur (Satan) un portrait de paria. A l'heure du remerciement, c'est un être reconnaissant qui cherche à renouveler sa confiance en Dieu.

Une analyse semblable peut bien entendu s'appliquer aux autres genres discursifs, mais nous ne développerons pas ce point ici, faute d'espace. Dans le genre «prophétie », le cadre figuratif présente invariablement Dieu en face des Fidèles et la fonction linguistique incitative est la plus prégnante. Dans les textes de «témoignage », par contre, le cadre figuratif place un fidèle face à d'autres fidèles, et le discours réfère itérativement à la méchanceté de Satan qui détruit et à l'amour de Dieu qui reconstruit; la fonction incitative est ici aussi prédominante. Quant au genre « enseignement », son cadre figuratif présente le pasteur (personne éclairée par Dieu) en face de son auditoire (fidèles ignorants) et ses énoncés sont surtout assertifs.

En définitive, les Eglises de réveil constituent un espace de circulation d'un discours religieux assez particulier et aux enjeux bien précis : le salut de l'âme, la santé du corps, la protection contre les mauvais esprits, le bien-être matériel et social des conducteurs et de leurs adeptes. Ce discours assume ainsi des fonctions messianique, prophétique et thérapeutique au sein de la société en crise. L'euphorie religieuse suscitée par ces sectes chrétiennes a profondément marqué les pratiques langagières des Kinois. Les mutations culturelles qu'elles ont introduites dans leur communauté de langage ont une incidence sociolinguistique certaine sur l'ensemble du christianisme; elles ont même marqué les pratiques langagières des Kinois qui attendent du ciel la nourriture quotidienne, un emploi rémunérateur, une protection contre les mauvais esprits, des bénédictions... Les Eglises de réveil détiennent donc tout un capital culturel qui impose son style langagier.

\section{Références bibliographiques}

Austin, J.-L., 1962. Quand dire c'est faire. Paris : Editions du Seuil.

Benveniste, E., 1966. Problèmes de linguistique générale 1. Paris : Gallimard.

Bronckart, J.-P., 1985. Le fonctionnement du discours. Un modèle psychologique et une méthode d'analyse. Bruxelles : PUB.

Matangila Ibwa, A., 2004. Les enjeux de la communication dans les Eglises de réveil à Kinshasa. Essai d'analyse d'un discours religieux en milieux diglossique. Thèse de doctorat, Faculté des lettres et sciences humaines. Kinshasa : UNIKIN.

SEARLE, J.-R., 1972 [1969]. Les actes de langage. Paris : Hermann. 